\title{
Electronic Energy Transfer In a Subphthalocyanine - Zn Porphyrin Dimer Studied by Linear and Nonlinear Ultrafast Spectroscopy
}

Giovanni Bressan ${ }^{1}$, Andrew N. Cammidge ${ }^{1}$, Garth A. Jones ${ }^{1}$, Ismael A. Heisler, ${ }^{2}$ Daniel GonzalezLucas, ${ }^{1}$ Sonia Remiro-Buenamañana ${ }^{1}$ and Stephen R. Meech*1

${ }^{1}$ School of Chemistry, University of East Anglia, Norwich NR4 7TJ

${ }^{2}$ Departamento de Física, Universidade Federal do Paraná, Caixa Postal 19044, 81531-990

Curitiba, Parana, Brazil

*corresponding author contact: s.meech@uea.ac.uk

\section{Abstract}

The efficient harvesting and transport of visible light by electronic energy transfer (EET) is critical to solar energy conversion in both nature and molecular electronics. In this work we study EET in a synthetic dyad comprising a visible absorbing subphthalocyanine (SubPc) donor and Zn Tetraphenyl Porphyrin (ZnTPP) acceptor. Energy transfer is probed by steady state spectroscopy, ultrafast transient absorption and two-dimensional electronic spectroscopy. Steady state and time resolved experiments point to only weak electronic coupling between the components of the dimer. The weak coupling supports energy transfer from the SubPc to the zinc porphyrin in $7 \mathrm{ps,} \mathrm{which} \mathrm{itself}$ subsequently undergoes intersystem crossing to populate the triplet state. The rate of the forward energy transfer is discussed in terms of the structure of the dimer, which is calculated by density functional theory. There is evidence of back energy transfer from the ZnTPP on the hundreds of picoseconds time scale. Sub picosecond spectral diffusion was also observed and characterised, but does not influence the picosecond energy transfer. 


\section{Introduction}

Understanding electronic excitation energy transfer (EET) in covalently bound molecular model systems gives insight into the behaviour of more complex artificial light harvesting structures ${ }^{1,2,3}$. Real time studies of the ultrafast photoinitiated dynamics taking place in model systems make it possible to pinpoint processes which either enhance or are detrimental to energy transport efficiency, allowing the design of new, more efficient, synthetic organic light harvesters for applications in, for example, organic photovoltaic (OPV) devices ${ }^{4,5,6,7}$. Among the different classes of organic molecules that could be employed as active materials in OPVs, porphyrins have attracted a great deal of attention because of their occurrence in biological systems, ${ }^{8,9}$ and because of their use in building supramolecular structures $^{10,11,12}$ and molecular nanowires displaying outstanding conductivity properties ${ }^{13}$ and as sensitisers in dye sensitised solar cells ${ }^{14,15}$. Porphyrins are also be used as sensitizers in photocatalytic applications $^{16,17}$. Their unique photophysical properties stem from an extended $18 \pi$-electron planar aromatic ring ${ }^{18}$. However, one of the main drawbacks in the use of porphyrins in solar energy applications is that their Q-band absorption is situated towards the red region of the visible spectrum, with a transition moment which is much smaller than the strong Soret (or B-) band absorption, usually lying between $400-450 \mathrm{~nm}^{19,20}$; thus the electronic structure leads to relatively poor utilization of the solar spectrum ${ }^{21}$. To circumvent this drawback, it is possible to link a porphyrin with an antenna chromophore absorbing light more effectively in the visible spectrum, and the subphtalocyanines studied here have been used as antennas for a range of acceptor chromophores. ${ }^{22,23}$ In this work we report the time-resolved photophysics of a heterodimer in which a light harvesting antenna, boron subphtalocyanine (SubPc), was linked through a phenoxy bridge at the meso position of a $\mathrm{Zn}$ (II) 5-(4hydroxyphenyl)-10,15,20-triphenylporphyrin (ZnTPP) (Figure 1). Other examples of axially linked SubPc-based structures have been synthesised and studied ${ }^{23-25}$, although, most of them present alkyne or alkene linkers, which present cylindrical or planar symmetry and are intrinsically rigid. Conversely, the dimer object of this study is axially liked to the ZnTPP acceptor via a flexible phenoxy bridge, which can substantially affect the rate and the efficiency of the energy transfer process. 


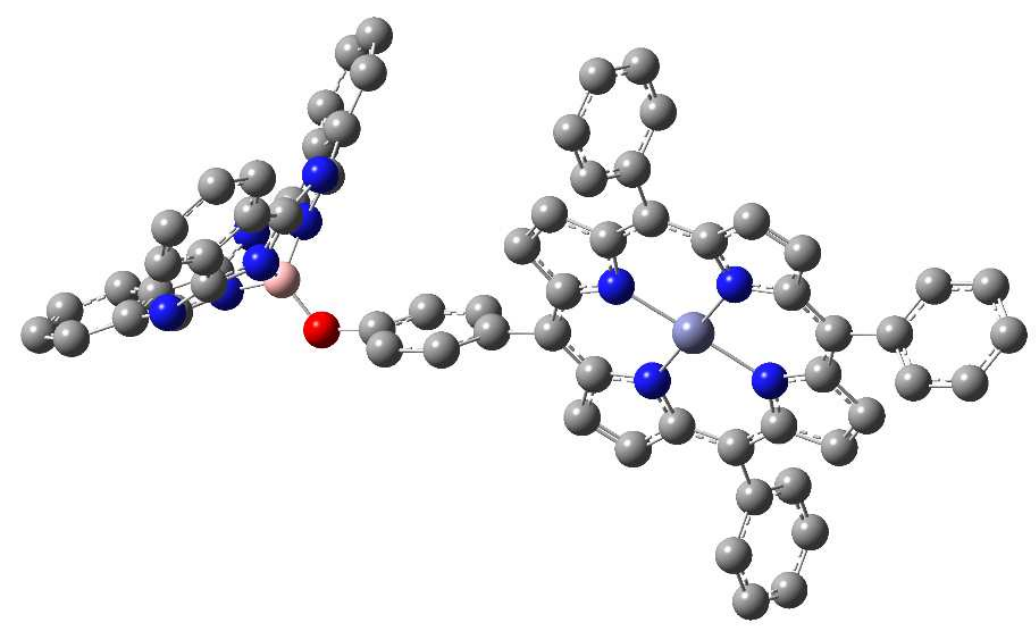

Fig. 1 DFT optimised structure of ZnTPP-O-SubPc calculated with B3LYP/6-31G(d) basis set.

SubPcs have a unique nonplanar cone-shaped $14 \pi$-electron aromatic system, whose excitation gives rise to intense absorption and emission bands in the visible region $\left(\lambda_{\max }=560 \mathrm{~nm}\right)^{26,27}$. Their spectral properties, together with their ease of functionalisation, both at the ring position and at the boron core ${ }^{28,29}$, make them promising candidates for optoelectronic (e.g. OLEDs) $)^{30,31}$ and photovoltaic applications ${ }^{32,3}$. In this work we investigate the photodynamics of the ZnTPP-O-SubPc heterodimer and its constituent monomers, through broadband femtosecond transient absorption (fsTA) and twodimensional electronic spectroscopy (2D-ES) experiments in toluene solution.

\section{Experimental Methods}

SubPc-Cl, ZnTPP-OH and ZnTPP-O-SubPc were synthesised and purified as previously reported ${ }^{33}$ and used without further purification.

Steady-state absorption was measured using a Lambda XLS Spectrophotometer (Perkin Elmer); data were collected in $1 \mathrm{~mm}$ quartz cuvettes in toluene at OD ca. 0.3. Steady-state emission spectra were measured with a fluorimeter (FLUOROSens, Camlin Technologies Ltd), with $1 \mathrm{~nm}$ excitation and $2 \mathrm{~nm}$ emission slits; emission spectra were acquired in $2 \mathrm{~nm}$ steps integrating each point for $0.1 \mathrm{~s}$, exciting 
at $530 \mathrm{~nm}$. Fluorescence spectra were measured in $10 \mathrm{~mm}$ quartz cuvettes with OD adjusted to ca. 0.1 to avoid concentration-induced effects.

The fsTA setup used is based on the design of Riedle ${ }^{34}$, and has been described elsewhere ${ }^{35}$. It employs an amplified titanium:sapphire laser system (Spitfire Ace, Spectra Physics) operating at $1 \mathrm{kHz}$, producing $120 \mathrm{fs}, 800 \mathrm{~nm}$ centred pulses. The laser output is split in two beams, one enters a commercial (TOPAS Prime) collinear optical parametric amplifier (OPA), tuned to produce $10 \mathrm{~nm}$ FWHM bandwidth pump pulses at $560 \mathrm{~nm}$. The reflected fraction of the $800 \mathrm{~nm}$ beam is attenuated and focused onto a static, $3 \mathrm{~mm}$ thick, sapphire plate for continuum generation, producing a probe spectrum spanning the $450-750 \mathrm{~nm}$ range. The pump is delayed with respect to the probe with a mechanical linear delay stage (Physik Instrumente). Pump and probe beams are focused with reflective optics at the sample position in order to obtain spot sizes of 170 and $30 \mu \mathrm{m}$ (FWHM) respectively, resulting in excitation energies below $0.4 \mathrm{~W} / \mathrm{cm}^{2}$. The pump beam is set the magic angle $\left(54.7^{\circ}\right)$ with a half-wave plate and a polariser to suppress effects of rotational reorientation; it is chopped at $500 \mathrm{~Hz}$ to allow calculation of differential absorption between adjacent pairs of pulses.

After the sample the probe beam is recollimated, focused onto a home-built ultrafast prism-based spectrometer and imaged on a CCD Camera (Stresing) triggered at $1 \mathrm{kHz}$ by the amplified laser; a 50:50 beamsplitter set before the sample holder sends half of the probe intensity through the same spectrometer and to a reference camera.

Equation 1 is used to compute the differential absorption, yielding negative signals for ground state bleach (GSB) and stimulated emission (SE) and positive signals for excited state absorption (ESA) or absorption by new products:

$$
\Delta O D(\lambda, t)=-\log \left(\frac{P r_{\text {odd }}(\lambda, t) R e f_{\text {even }}(\lambda)}{P r_{\text {even }}(\lambda) \operatorname{Ref} f_{\text {odd }}(\lambda)}\right)
$$


where $\operatorname{Pr}(\lambda)$ and $\operatorname{Ref}(\lambda)$ are probe and reference spectra and the pump is being blocked by the chopper during the acquisition of even data points. To correct for chirp in the white light probe, a TA measurement on neat cyclohexane was run under the same experimental condition as the sample. The solvent coherence spike maxima as a function of probe wavelength are fit with a fourth order polynomial, and the resulting time vs wavelength profile is subtracted from the raw dataset. The fsTA data presented in the Results section are the average of 8 scans, in which 500 differential spectra were averaged at each delay point.

The design and the data acquisition procedures for the 2D-ES setup were described in detail elsewhere ${ }^{36}$. Briefly, a fraction ( $\left.10 \%\right)$ of the same amplified laser system used for the fsTA experiment pumps a commercial (TOPAS White, Light Conversion) noncollinear optical parametric amplifier (NOPA) tuned to generate $\sim 15$ fs pulses centred at $560 \mathrm{~nm}$ with ca. $30 \mathrm{~nm}$ FWHM. The pulses are recompressed close to the Fourier transform limit with a folded prism compressor and characterised using the 2D setup, via a transient grating-frequency resolved optical gating (TG-FROG) signal from a $1 \mathrm{~mm}$ quartz cell filled with cyclohexane. The output of the NOPA is split into four coherent replicas propagating at the four corners of a $2.5 \mathrm{~cm}$ square (BOXCARS scheme), by means of two $50-50$ beamsplitters. The coherence time (between pulses 1 and 2$)(\tau)$ is scanned with a mechanical delay stage in $4 \mathrm{fs}$ steps from -150 to $+150 \mathrm{fs}$ and population times (between pulses 2 and 3 ) (T) up to 600 ps can be measured. After the interaction of pulses 1,2 and 3 with the sample a third order signal, collinear with the LO beam is emitted in the phase matched direction. The interference of signal and local oscillator (LO) is dispersed with a prism and imaged onto a $1 \times 1024$ pixels fast camera (AViiVa e2v). Spectral interferometry allows us to extract amplitude and phase of the signal, which is phased according to the projection-slice theorem ${ }^{37}$, while the time delay between pulses 1 and 2 is Fourier transformed to recover the excitation axis. 


\section{Results}

\section{Steady-state absorption and emission}

The steady-state normalised absorption and emission spectra (excitation at $530 \mathrm{~nm}$ ) of the two monomers SubPc-Cl and ZnTPP-OH are shown in Figure 2a and ZnTPP-O-SubPc dimer spectrum is shown in Figure 2b. All spectra measured in in toluene.

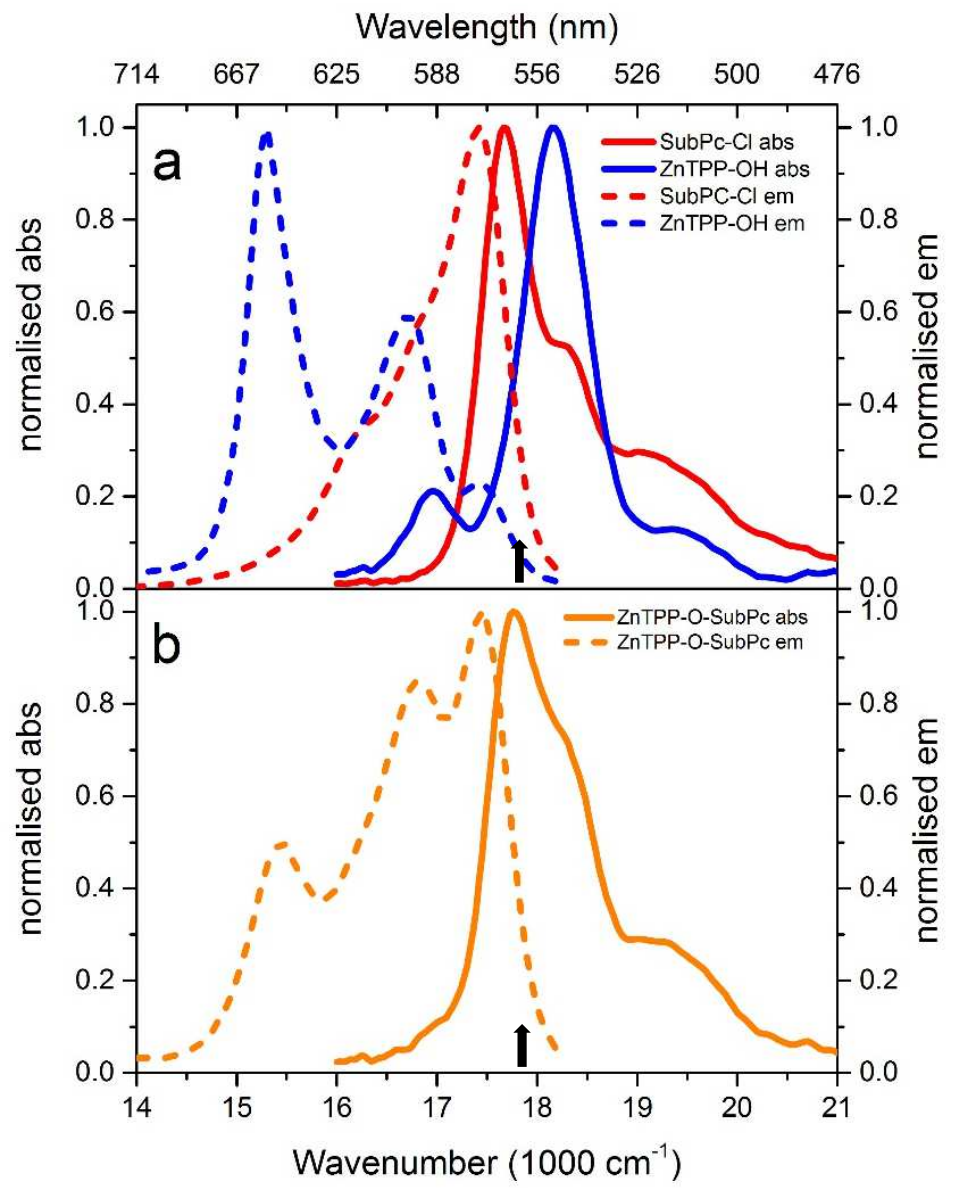

Fig. 2 Normalised absorption (solid) and emission (dashed) spectra of ZnTPP-OH (a, blue), SubPc-Cl (a, red) and ZnTPP-OSubPc (b, orange). The central pump wavenumber for fsTA and 2D-ES $(560 \mathrm{~nm})$ is highlighted by black solid arrows. Emission spectra measured after excitation at $530 \mathrm{~nm}$.

This dimer system was previously studied through steady state experiments and calculation ${ }^{33}$. The absorption and emission spectra of the ZnTPP-O-SubPc were shown to resemble the sum of the SubPc$\mathrm{Cl}$ and ZnTPP-OH spectra, pointing to a weakly coupled system with negligible perturbation of the electronic structure of the two units as anticipated by Managa et $a l^{33}$. 
The absorption spectrum of SubPc-Cl (Figure 2a) has a maximum centred at $17668 \mathrm{~cm}^{-1}(566 \mathrm{~nm}$ ) due to the $0-0$ transition of the Q-band, as reported in literature ${ }^{32}$; the vibronic progression matches well the spectra reported in literature. The emission spectrum, is a quasi-mirror image of the absorption, with a maximum at $17421 \mathrm{~cm}^{-1}(574 \mathrm{~nm})$ and the fluorescence quantum yield is $0.25^{32}$. The small Stokes shift of $247 \mathrm{~cm}^{-1}(8 \mathrm{~nm})$, indicates a minor effect of the solvent after photoexcitation of the sample, as expected for nonpolar solvents, or when the change in permanent dipole between ground and excited state is small.

The main peaks in the steady-state absorption of ZnTPP-OH (Figure $2 a$ ) are the vibronic $Q(1,0)$ band, centred at $18148 \mathrm{~cm}^{-1}(551 \mathrm{~nm})$, and the weaker and red-shifted $\mathrm{S}_{0} \rightarrow \mathrm{S}_{1} \mathrm{Q}(0,0)$ transition, at $16949 \mathrm{~cm}^{-}$ ${ }^{1}(590 \mathrm{~nm})$. The steady-state fluorescence spectrum of ZnTPP mirrors the absorption in terms of peak positions, $Q(0,0)$ is at $16680 \mathrm{~cm}^{-1}(600 \mathrm{~nm})$ and $Q(1,0)$ lies at $15302 \mathrm{~cm}^{-1}(653 \mathrm{~nm})$ with a $Q(0,1)$ "hotband" shoulder at $17391 \mathrm{~cm}^{-1}(574 \mathrm{~nm})$ making a significant contribution to the emission; these measurements are in good agreement with previously reported data on the same molecule ${ }^{20}$. The measured Stokes shift between the absorption and emission bands of the $Q(0,0)$ transition is $270 \mathrm{~cm}^{-}$ ${ }^{1}(10 \mathrm{~nm})$. ZnTPP-OH is only weakly fluorescent because of strong spin-orbit coupling due to the presence of a heavy metal ion; its fluorescence quantum yield in toluene was reported to be $0.03^{38}$.

In the ZnTPP-O-SubPc dimer steady-state absorption spectrum (Figure $2 \mathrm{~b}$ ) no major new features are observed, consistent with weak coupling between the subphtalocyanine and porphyrin units. However, the absorption maximum is blue-shifted $\sim 350 \mathrm{~cm}^{-1}$ with respect to the SubPc monomer (which is assigned to the different substituent at the boron core: $-\mathrm{Cl}$ in the monomer, -OPh in the dimer), and the overall band is broader, indicative of the overlap of the SubPc and ZnTPP Q -bands. The dimer steady-state emission spectrum is a combination of the fluorescence spectra of the SubPc$\mathrm{Cl}$ and $\mathrm{ZnTPP}-\mathrm{OH}$ monomers, but the intensity of the $\mathrm{Zn}$ porphyrin contribution appears enhanced compared to what is expected from absorbance (Figure 3), while the overall signal is quenched, as shown in Figure S2 (see Supplementary Information). 
This apparent change in the relative emission intensities of SubPc and ZnTPP is an indication of EET. As a check we calculated the expected emission spectrum of the dimer assuming no energy transfer between the singlet excited states of SubPc-Cl and $\mathrm{ZnTPP}-\mathrm{OH}$, and compared it to the measured dimer spectrum (Figure 3). The dimer emission spectrum was calculated as explained in the Supplementary Information.

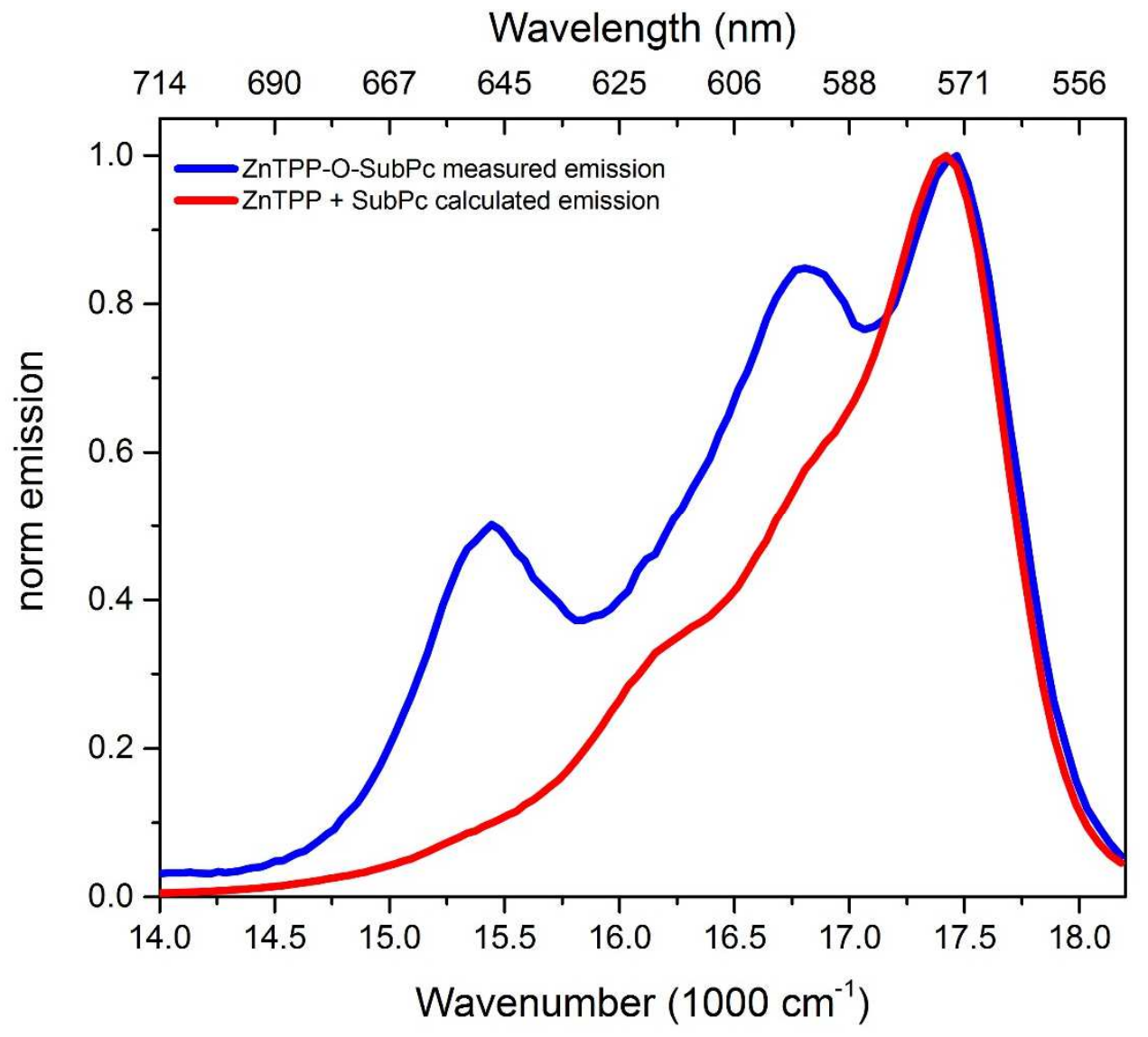

Fig. 3 Comparison between the ZnTPP-O-SubPc calculated emission spectrum (red) and the experimentally measured one (blue).

\section{Excitation Energy Transfer (EET) dynamics from SubPc antenna to ZnTPP in toluene}

The results from the steady state emission suggest that the singlet excited state of SubPc and the ZnTPP moieties in the dyad are weakly coupled, supporting Förster Resonance Energy Transfer (FRET). To better characterise the rate and mechanism of EET, broadband fsTA measurements were carried 
on the dimer and its constituent monomers in toluene at room temperature, with pump wavelength fixed at $560 \mathrm{~nm}$.

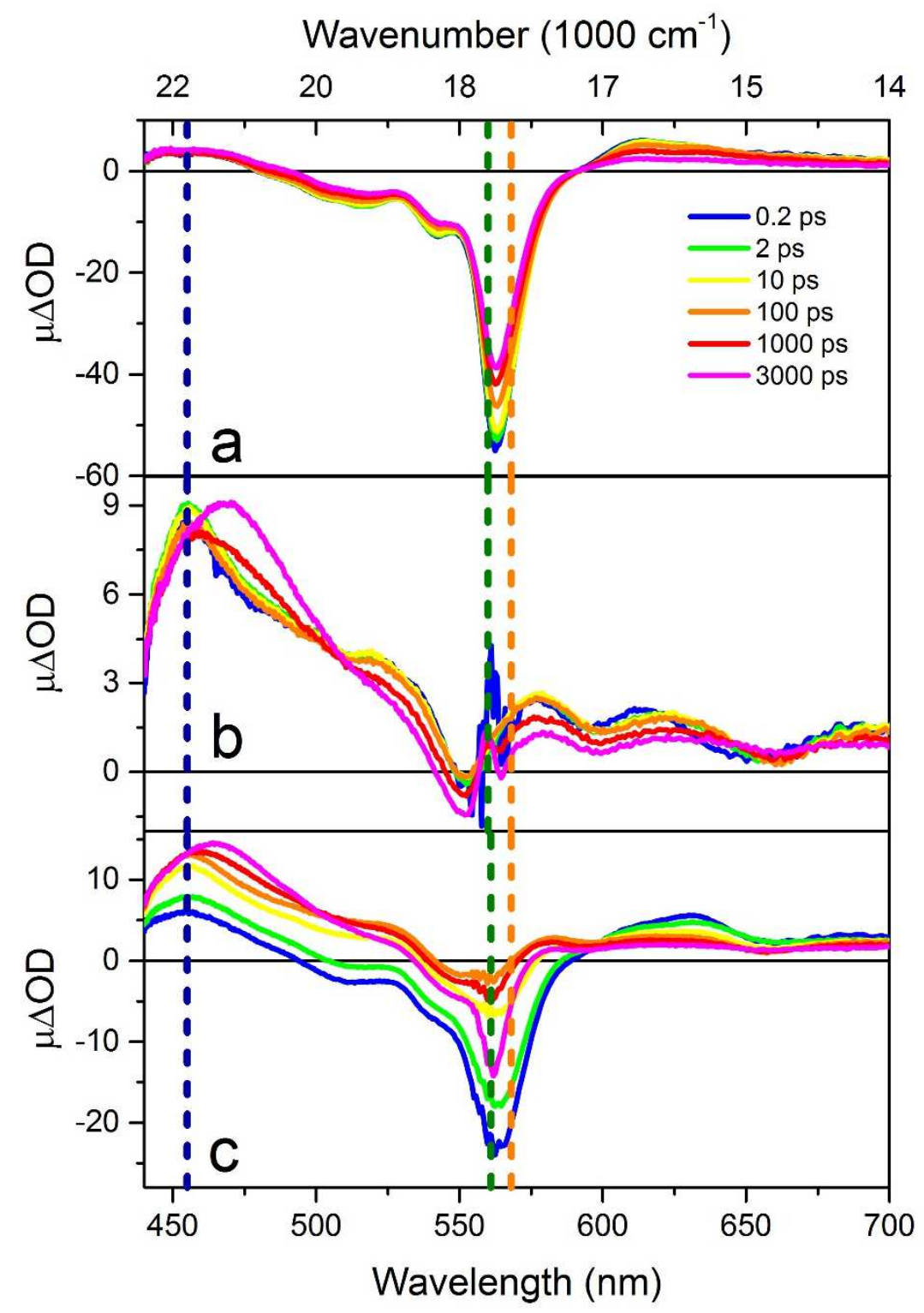

Fig. 4 fsTA spectra of SubPc-Cl (a), ZnTPP-OH (b) and ZnTPP-O-SubPc (c) in toluene at selected pump-probe time delays, after photoexcitation at $560 \mathrm{~nm}$ (green dashed line). Notice that the pump scattering contribution looks intense in (b) because of the small amplitude of these signals, compared to (a) and (c). Dark blue and orange dashed lines at $455 \mathrm{~nm}$ and $568 \mathrm{~nm}$ highlight the wavelength at which the intensity-time traces shown in Fig. 5 are presented.

The SubPc-Cl transient spectra at selected pump-probe delays are reported in Figure 4a. The earliest transient spectrum ( $0.2 \mathrm{ps}$, earlier spectra being somewhat distorted by the coherence spike) has a strong negative signal, arising from a combined effect of the GSB, corresponding with the steady-state absorption spectral maximum (566 nm), and the SE component, centred at the same spectral position as the steady-state fluorescence maximum $(574 \mathrm{~nm})$. Two broad positive excited state absorption 
(ESA) $S_{1} \rightarrow S_{n}$ signals are centred at $460 \mathrm{~nm}$ and $610 \mathrm{~nm}$. The subsequent spectral evolution of SubPc TA is straightforward to explain in terms of simple population decay of the $S_{1}$ state, accompanied by formation of a $T_{1}$ state via intersystem crossing (ISC) on the ns timescale, as shown by the decay of the broad $\mathrm{S}_{1} \rightarrow \mathrm{S}_{\mathrm{n}}$ ESA which is peaked at $610 \mathrm{~nm}$ and elongated towards the red region of the spectrum. Even at the longest experimentally measurable pump-probe delay time ( $3 \mathrm{~ns})$ the bleach has not recovered and the TA persists ( $\tau_{\text {fluo }}$ lifetime reported to be $2.1 \mathrm{~ns}^{39}$ ). The triplet state lifetime for a related SubPc-Cl was reported to be in the microsecond range ${ }^{40}$.

Figure $4 \mathrm{~b}$ shows the transient spectra of $\mathrm{ZnTPP}-\mathrm{OH}$ after excitation at $560 \mathrm{~nm}$. At $0.2 \mathrm{ps}$, at least four ESA $\left(S_{1} \rightarrow S_{n}\right)$ bands in the transient spectrum are observed, some of them are partially overlapped with a weak GSB signal, corresponding to the steady-state maximum of the absorption spectrum (551 $\mathrm{nm})$. The overall signal is weak because ZnTPP is being excited at a spectral position where it is weakly absorbing; in order to make the fsTA measurements of the monomer directly comparable to the excitation for the heterodimer the excitation wavelength was kept fixed for all the samples. Interestingly, the signal experiences a rise in the strongest ESA $(455 \mathrm{~nm})$ region between $0.2 \mathrm{ps}$ and 2 ps, followed by slower population decay within the first ns after photoexcitation. In the two latest spectra measured (1-3 ns) the most intense positive signal is red shifted ( $468 \mathrm{~nm}$ ) with respect to the singlet ESA, assigned to the formation of a triplet state through ISC $\left(\tau_{\text {fluo }} \approx 1.8 \mathrm{~ns}^{41}\right)$ which then has a distinct ESA $\left(T_{1} \rightarrow T_{n}\right)$ spectrum ${ }^{42}$. The occurrence of ISC is consistent with the GSB not recovering, while the $S_{1} \rightarrow S_{n}$ singlet ESA peaks $(575,620$ and $691 \mathrm{~nm})$ experience a partial decay to leave a signal, also assigned to the triplet state.

When the ZnTPP-O-SubPc dimer is excited at $560 \mathrm{~nm}$ (Figures 4c and 5) a mixture of signals ascribable to the excitation of the two moieties is detected at $0.2 \mathrm{ps}$, as expected from their common absorption at $560 \mathrm{~nm}(\mathrm{ESI})$. However, the spectral evolution of the TA is remarkably different from either of the two monomers. In this case, within the first 10 ps the negative GSB + SE of SubPc centred at $555 \mathrm{~nm}$ signal loses $\sim 80 \%$ of its initial amplitude, while the positive ESA ZnTPP signal at $455 \mathrm{~nm}$ experiences a 
threefold rise within the same timescale (orange and blue triangles in Figure 5). The SubPc ESA centred at $630 \mathrm{~nm}$ decays in the same way as the negative features recovers.

The difference in dynamics within the first 10 ps is consistent with EET between the electronically excited SubPc antenna and the ZnTPP acceptor. On this timescale the excitation moves from the $\mathrm{S}_{1}$ excited state of the subphthalocyanine to create a population in the $S_{1}$ state of the $\mathrm{Zn}$ porphyrin; once the ZnTPP singlet state is populated, ESA to its higher energy singlet states is observed.

At longer pump-probe delays, the overall signal decays/recovers via population relaxation dynamics, and the ZnTPP triplet state is formed on the ns timescale. The increasingly negative GSB at $568 \mathrm{~nm}$ observable in the latest transient spectrum ( $3 \mathrm{~ns}$, Figure 4c) and in the long-time behaviour of the orange triangles shown in Figure 5 is unexpected. This effect is too large to be simply assigned to the disappearance of the broad underlying singlet ESA (from 500 to $630 \mathrm{~nm}$ ) of the ZnTPP (which may be sufficient to explain the small effect on the monomer in $4 b$, as discussed in $\$ 4)$, but rather appears to be a true bleach on the nanosecond timescale. We speculate that this may indicate a reverse energy transfer step. The superimposed magenta traces are extracted from the global fitting procedure which will be described in the Discussion.

Intramolecular vibrational relaxation (IVR), vibrational cooling and solvent dynamics, all occur on the order of, or faster than, EET and can therefore affect its dynamics, kinetics and efficiency. Indeed there are hints in the TA data (e.g. the initial fast relaxation of SubPc-Cl and ZnTPP, Figures $4 a, b$ and 5) that suggest these contributions could play a role. The detection of such processes with fsTA measurements is challenging, because of the spectral congestion and limited time resolution of the experiment. For these reasons, we carried out two-dimensional electronic spectroscopy (2D-ES) experiments on the ZnTPP-O-SubPc dimer and its constituent moieties. 


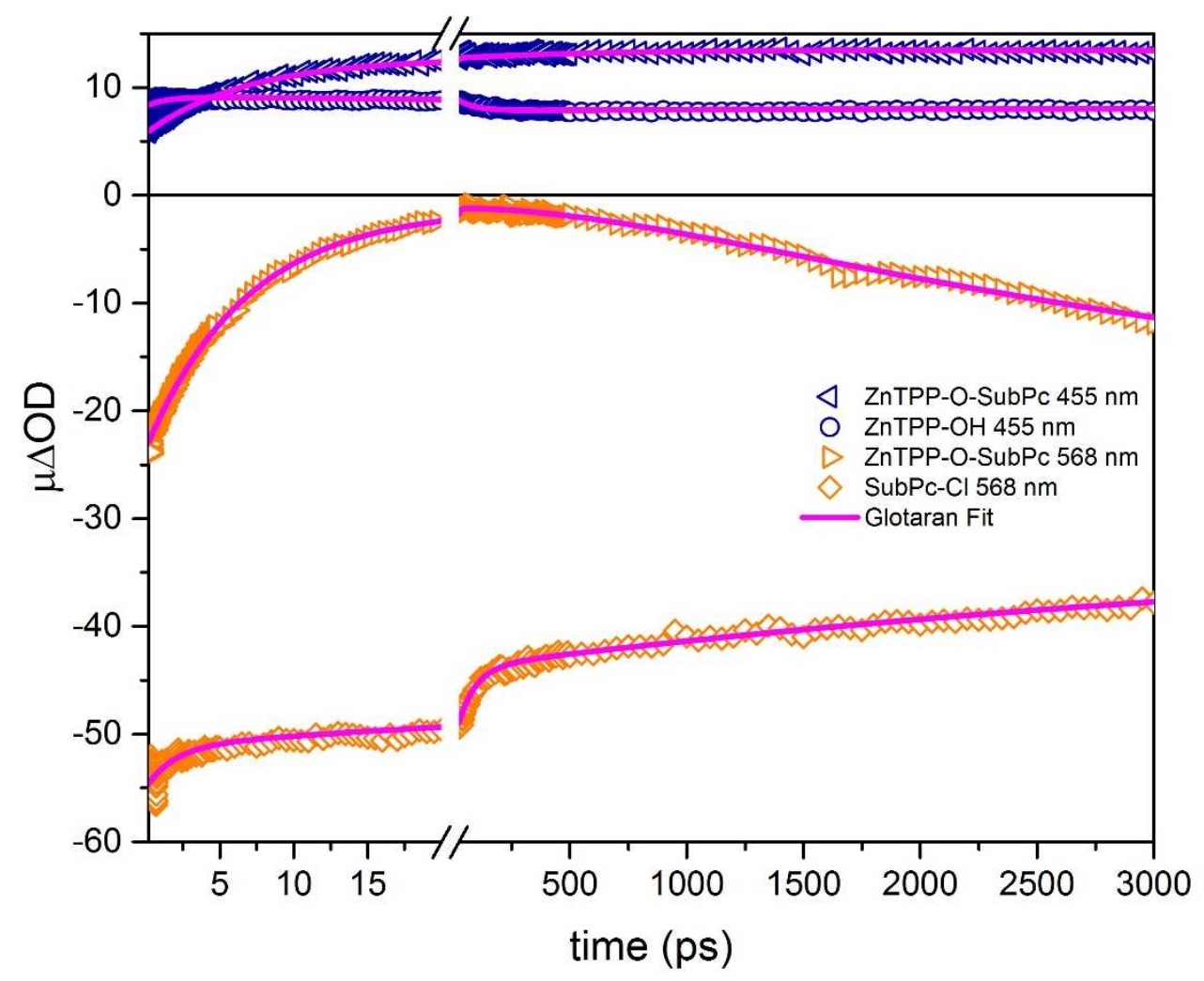

Fig. 5 pump-probe time delay vs $\mu \triangle O D$ traces at $455 \mathrm{~nm}$ (dark blue) and $568 \mathrm{~nm}$ (orange) for SubPc-Cl (diamonds), ZnTPP$\mathrm{OH}$ (circles) and ZnTPP-O-SubPc (triangles), overlaid with traces of the global fit of the corresponding dataset at the same wavelength (magenta). The break on the horizontal axis highlights the EET dynamics, happening within the first 20 ps.

\section{Sub-Picosecond dynamics by 2D-ES}

2D-ES measurements were carried at room temperature in toluene, using $30 \mathrm{~nm}$ FWHM pulses from a NOPA centred at $560 \mathrm{~nm}$, in order to cover both the Q-band transition of the ZnTPP and the SubPc absorption. 2D-ES data were measured up to $T=600 \mathrm{ps}$ and clearly showed the EET discussed in the preceding section (see $\mathrm{SI}$ ), here we focus on the spectral reshaping and dynamic Stokes shift, as signatures of vibrational cooling and/or solvent rearrangement on a sub-ps timescale.

A series of absorptive (sum of the real part of the rephasing and nonrephasing signals) 2D spectra for SubPc-Cl and ZnTPP-O-SubPc are shown in Figure 6 left and right, respectively. The 2D data on ZnTPP$\mathrm{OH}$ monomer are not relevant in this context because we focus on the reshaping and red shift of the positive signal in ZnTPP-O-SubPc, which is dominated by the subphthalocyanine contribution in the spectral region interrogated. The spectral progression in the same time window of $\mathrm{ZnTPP}-\mathrm{OH}$ is shown in the SI for completeness. 

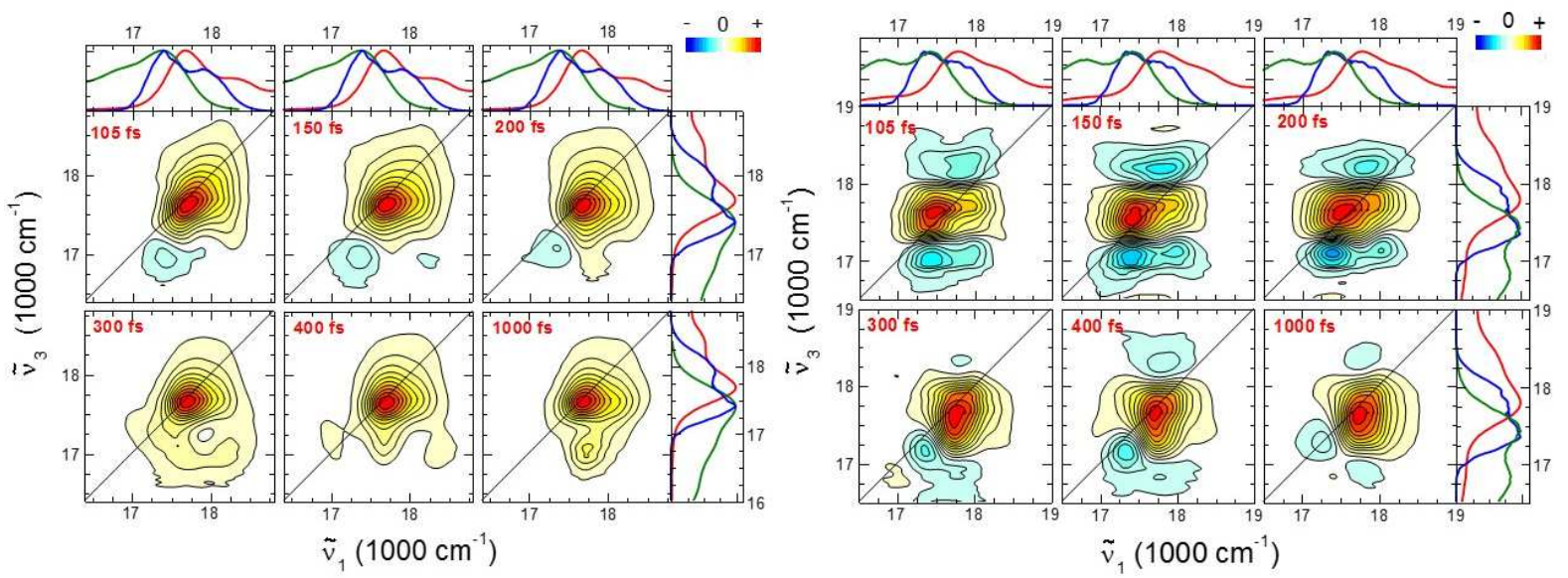

Fig. 6 Absorptive 2D-ES spectral progression for SubPc-Cl in toluene (left) and ZnTPP-O-SubPc (right), where $v_{1}$ is the excitation and $v_{3}$ is the detection frequency (plotted in wavenumber). Each 2D graph is normalised to its positive maximum and the amplitude has 21 evenly spaced contour lines. Positive signals are coloured in yellow or red, and represent Ground State Bleaches or Stimulated Emission pathways, while negative signals are coloured in blue and represent Excited State Absorption pathways. Red numbers on the top left of each map indicate the population time $T$ and in the top and right panel the steady-state absorption (red), emission (green) and NOPA spectra (blue) are shown.

At the earliest population time SubPc-Cl shows mixed positive GSB and SE signal, peaked at the steady state absorption and emission maxima $\left(\tilde{v}_{1}=17712 \mathrm{~cm}^{-1}, \tilde{v}_{3}=17642 \mathrm{~cm}^{-1}\right)$. The signal is elongated along the diagonal, a clear signature of inhomogeneous broadening of the electronic transition at early population times ${ }^{43}$. A weak negative off-diagonal $\left(\tilde{v}_{1}=17398 \mathrm{~cm}^{-1}, \tilde{v}_{3}=16953 \mathrm{~cm}^{-1}\right)$ ESA peak is present as well. Intramolecular vibrational and solvent relaxation occur in the $\mathrm{S}_{1}$ state, which translates as a reshaping of the positive 2DES signal within the first ps, this effect can be quantified by extracting and analysing the centre line slope ${ }^{44,45}$ (CLS) of the 2D data, as shown in the Discussion (below).

The fast disappearance ( $200 \mathrm{fs}$ ) of the ESA peak (Figure 6, left) can be explained in terms of relaxation in the $S_{1}$ potential energy surface (PES): the initially prepared "hot" population in the $S_{1}$ state manifold can absorb a second photon to give $S_{1} \rightarrow S_{n} E S A$, but once the relaxation of the hot population occurs the third NOPA pulse does not have sufficient bandwidth to be resonant with the relaxed ESA. The blue-shift on the detection axis of the negative peak is consistent with this hypothesis.

For the ZnTPP-O-SubPc at early population times $(T=105-200 \mathrm{fs}$, Figure 6 , right $)$ the positive GSB + SE 2D spectra display one maximum centred above the diagonal $\left(\tilde{v}_{1}=17489 \mathrm{~cm}^{-1}, \tilde{v}_{3}=17619 \mathrm{~cm}^{-1}\right)$, matching with the steady state absorption and emission, as observed in the SubPc-Cl monomer. The 
overall signal is broader, which we speculate might be due to increased flexibility of the dimer, which can explore a wider range of conformations both in the ground and in the excited state PESs. The initial broad structure stretched along the diagonal, after 200 fs evolves towards a more elliptical shape, while the maximum is shifting to lower detection frequency. This effect (which is consistent with a dynamic Stokes shift) can be assigned to a relaxation of the $S_{1}$ state, alongside spectral diffusion, as confirmed by the CLS analysis (see Discussion).

The predominant feature of the dimer early population times spectra (Figure 6, right, top row), is the presence of two intense negative ESA signals assigned to the ZnTPP, matching with the fsTA behaviour of the isolated ZnTPP monomer and of the dimer. The main effect of those negative signals is to alter the shape of the positive signal, making it more challenging to quantify spectral diffusion and dynamic Stokes shift from the 2D data of the ZnTPP-O-SubPc dimer.

\section{Discussion}

\section{EET dynamics in toluene}

The fsTA data for monomers and dimer (Figures $4 a, b, c$ and 5) were analysed with a global fitting procedure, in which the time-dependent difference spectra (as a function of the pump-probe delay) were fit to a single model incorporating a sum of exponential relaxations ${ }^{45,46}$. This model assumes a parallel decay mechanism, and the exponential amplitudes retrieved are called Decay Associated Difference Spectra (DADS, shown in the SI, Figure S3). An equivalent but sequential model, yielding evolution associated difference spectra (EADS), in which the initial spectrum is evolving in successive first order steps to the product, can also be assumed, and is more appropriate for the EET dynamics observed here. EADS are also included for the monomers for the purpose of the comparison with the data in Figure 4. The dynamics in the SubPc and ZnTPP isolated monomers reflects a non-single exponential decay better described through DADS. Consequently, the parallel decay scheme yielding DADS for each time constant is discussed in the SI (S3). The resulting Evolutionary Associated Difference Spectra (EADS) are calculated from the DADS and are shown in Figure 7a for SubPc-Cl, 7b 
for ZnTPP-OH and 7c ZnTPP-O-SubPc dyad. To adequately fit the data required a model with three steps, yielding a final spectrum.

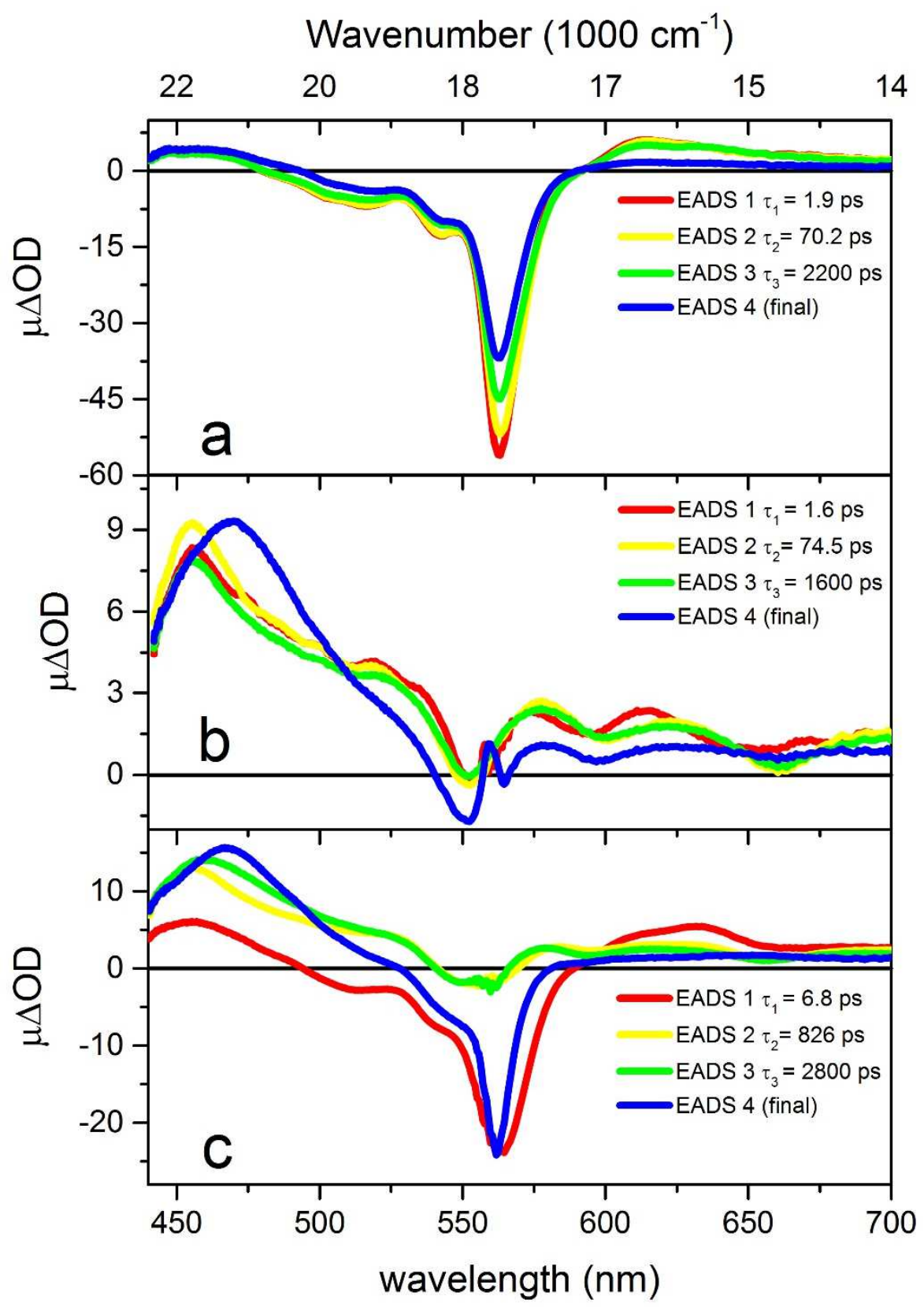

Fig. 7 Global analysis EADS spectra extracted from 4 component fit of SubPc-Cl (a), ZnTPP-OH (b) and ZnTPP-O-SubPc (c). EADS 1, 2, 3 and the final spectrum are respectively plotted in red, yellow, green and blue. The time constants associated with each spectrum are summarised in Table 1.

The EADS of the SubPc-Cl TA all show essentially the same shape, consistent with the domination of singlet state decay (Figure 7a). The earliest shows an initial fast (1.9 ps, red) relaxation that can be assigned to intramolecular vibrational energy redistribution (IVR) and vibrational cooling (VC) in the excited state; solvent dynamics may also occur on this timescale, but should be less significant in a 
nonpolar solvent such as toluene. The second shortest time constant (70 ps, yellow) has a similar spectrum to the 1.9 ps component. This intermediate lifetime was required to accurately fit the data, although this timescale is not compatible with any vibrationally-driven decay processes, and we were not able to assign it to any specific molecular relaxation mechanism. It does not lead to any new spectral features, so suggests multiexponential population relaxation in the isolated SubPc-Cl. The 2.2 ns spectrum (green) is associated with the decay of the singlet excited state (SubPc has a previously reported $\mathrm{S}_{1}$ fluorescence lifetime of $2.1 \mathrm{~ns}^{39}$ ) resulting in partial recovery of the GSB and loss of amplitude in the $S_{1} \rightarrow S_{n}$ absorption at $620 \mathrm{~nm}$. The final spectrum (blue), is the assigned to populations of the SubPc triplet state. There is a broad triplet ESA centred at $470 \mathrm{~nm}$ and elongated towards the red end of the spectrum ${ }^{40}$.

The same global fitting procedure allows us to extract time constants, DADS (shown in the SI) and EADS of the ZnTPP monomer excited at $560 \mathrm{~nm}$ (Figure $7 \mathrm{~b})$. Its fast (1.5 ps, red) initial decay is assigned to IVR or VC dynamics, which may include relaxation from two-photon excited higher electronic states, since the cross section of two-photon transition to the N-band of ZnTPP is known to be exceptionally large ${ }^{41}$. The 75 ps component (yellow) has a spectrum substantially equivalent to the $1.6 \mathrm{~ns}$ EADS, so again probably reflects a non-single exponential excited state decay.

The aforementioned 1.6 ns component (green) corresponds to the decay of the singlet excited state of the $\mathrm{Zn}$ porphyrin (literature fluorescence lifetime is $1.8 \mathrm{~ns}^{41}$ ). The final spectrum can be assigned to the ZnTPP triplet, the $\mathrm{S}_{1}$ ESA at $460 \mathrm{~nm}$ and between 570 and $720 \mathrm{~nm}$ has decayed and a new broad and intense triplet ESA at $471 \mathrm{~nm}$ is detected (triplet-triplet ESA for ZnTPP in toluene is reported elsewhere at $474 \mathrm{~nm}^{42}$ ) with apparently weaker features on the red side. An apparent deepening of the GSB at $551 \mathrm{~nm}$ is present, but it can be explained in terms of relaxation of the underlying broad singlet ESA signal.

The global fit of the dimer dataset also required four EADS (Figure 7c). The fastest process is assigned to the SubPc to ZnTPP EET (6.8 ps, red), because it shows strong negative signals comparable to the 
transient spectra of the SubPc, and the simultaneous presence of the $460 \mathrm{~nm}$ and $620 \mathrm{~nm}$ ESA of the $\mathrm{S}_{1}$ state of the ZnTPP which is being populated. The second EADS (yellow, $826 \mathrm{ps)}$ ) is dominated by combined population decay of the excited singlet states of ZnTPP and of the residual population left in the $S_{1}$ state of SubPc, which may arise from a slow (100s of ps) reverse energy transfer taking place from the $S_{1}$ state of the ZnTPP to the $S_{1}$ of the SubPc (see below). The second longest EADS spectrum (green, $2.8 \mathrm{~ns}$ ) matches closely the one associated with the 826 ps time constant (yellow), apart from a rise on the red side of the ESA peaked at $460 \mathrm{~nm}$. By comparison with the ZnTPP EADS it is reasonable to assign this component to the ISC of ZnTPP. The final spectrum (blue) reflects the unexpected disappearance (increasing bleach) for the SubPc spectrum at longer times noted in the experimental data (Figures $4 \mathrm{c}$ and 5).

Table 1: Time constants determined from the global analysis of the fsTA data.

\begin{tabular}{llll}
\hline & SubPc-Cl & ZnTPP-OH & ZnTPP-O-SubPc \\
\hline$\tau_{1}$ (ps) & 1.9 & 1.6 & 6.81 \\
\hline$\tau_{2}$ (ps) & 70.2 & 74.5 & 826 \\
\hline$\tau_{3}$ (ps) & 2200 & 1600 & 2800 \\
\hline
\end{tabular}

The experimentally recovered 6.8 ps relaxation time for the EET process may be compared to the calculated value, using the Förster Resonant Energy Transfer (FRET) model (Equation 5)

$$
k^{F R E T}=\frac{\frac{1}{\tau_{D}} 9 \ln (10) \kappa^{2} \varphi_{D} J^{F}}{128 \pi^{5} N_{A} n^{4}} \frac{1}{R^{6}}
$$

In which $\tau_{D}$ is the SubPc (donor) lifetime in the absence of ZnTPP (acceptor) (2.1 ns), $\kappa^{2}$ is a factor depending on the relative orientations of donor and acceptor ${ }^{48}, \varphi_{D}$ is the SubPc-Cl fluorescence quantum yield in the absence of acceptor $(0.25), J^{F}$ is the overlap integral between the emission 
spectrum of SubPc and the absorption spectrum of ZnTPP $\left(4.62 \times 10^{-14} \mathrm{M}^{-1} \mathrm{~cm}^{-1} \mathrm{~nm}^{4}\right), n$ is the refractive index of the solvent, in this case toluene $(n=1.5)$ and $R$ is the center-to-center distance, taken as the distance between the boron atom in SubPc and the zinc atom in ZnTPP (10.1 $\AA$ ), determined by the DFT calculation of the ground state of the dimer, including a dielectric continuum which accounts for the solvent shielding effects (Figure 8).

The $\kappa^{2}$ parameter was calculated with the help of DFT. The ground state geometry was optimized using the hybrid functional B3LYP employing the 6-31G* basis set. A harmonic frequency analysis of the minimised energy structure showed that it was a genuine minimum with no imaginary frequencies. See SI for Cartesian coordinates of the optimized geometry. There is the potential for rotation around three single bonds in the optimized structure, namely C-C, B-O and C-O (labelled R1, R2 and R3 in Figure 8). Constrained optimizations were run about each of these torsional degrees of freedom to map the potential energy surface along each coordinate. Figure 8 shows that all three barriers exceed $k T$ at $300 \mathrm{~K}(\mathrm{ca} .2 .5 \mathrm{~kJ} / \mathrm{mol})$ : Rotation about C-O has the lowest barrier at $3.4 \mathrm{~kJ} / \mathrm{mol}$, rotation about $\mathrm{O}-\mathrm{B}$ is ca. $5.0 \mathrm{~kJ} / \mathrm{mol}$, with the barrier to rotation for C-C being very high, due to steric clashes between the protons on the phenoxy bridge and those on the porphyrin.
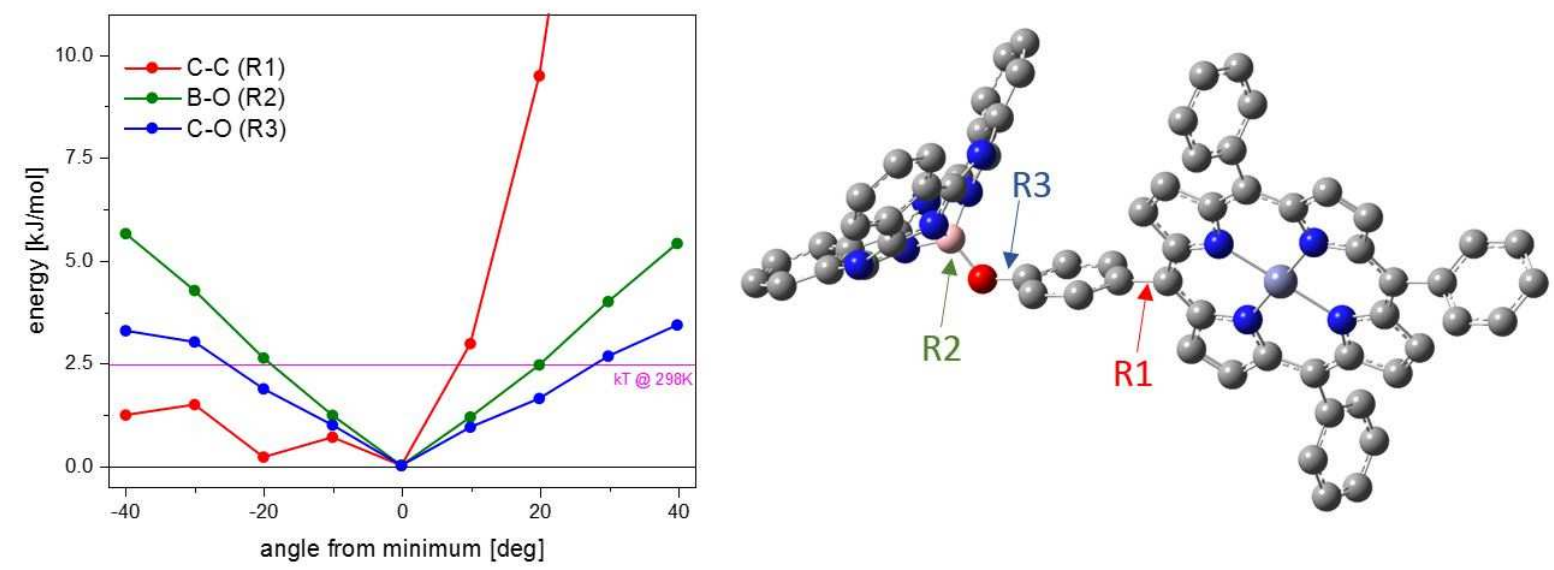

Fig. 8 Potential energy vs angle from their minimum energy structure for rotations around the $C-C(R 1, r e d), B-O$ (R2, green) and $C-O(R 3$, blue) in ZnTPP-O-SubPc. The value of $k T$ at $298 \mathrm{~K}$ is shown as a horizontal magenta line. All energies obtained from DFT calculations (left). Ground state DFT minimized energy structure for ZnTPP-O-SubPc (protons hidden for clarity) in which the bonds around which the rotations were calculated are highlighted with arrows and labelled (right). 
Consequently, we assume $\kappa^{2}$ can best be calculated at the optimized ground state geometry. To do this, calculations of the individual chromophores were performed at the same level of theory by first performing ground state optimizations followed by single point TDDFT calculations to extract transition dipole moments. Both chromophores possess two degenerate $S_{0} \rightarrow S_{1}$ transition dipole moments, shown in Figure S8 (see Supplementary Information). Their vectors were summed and the resultant dipole moments for each species, along with the displacement vector defined by the Boron and Zinc centres was used to calculate the $\kappa^{2}$ value, which yielded a value of 0.218 .

In principle, the orientation factor can take a range of values between 0 - 4 depending on the relative orientation of the donor and acceptor transition dipole moments. Consequently, the dimer of this study has a geometry yielding a value at the lower end of this range. In the dynamic averaging condition limit, satisfied if SubPc and ZnTPP dipoles were sampling all the possible orientations, the average value of $\kappa^{2}=0.444$, which will give a $k_{\text {calc }}^{F R E T}=3.82 \times 10^{11} s^{-1}\left(\tau_{\text {calc }}^{F R E T}=2.62 p s\right)$, significantly larger than the experimentally measured rate. Using Equation 5 and the minimum energy geometry we obtained a $k_{\text {calc }}^{\text {FRET }}=1.88 \times 10^{11} \mathrm{~s}^{-1}\left(\tau_{\text {calc }}^{F R E T}=5.32 \mathrm{ps}\right)$, which is in satisfactory agreement with the experimental rate $k_{\text {exp }}^{F R E T}=1.47 \times 10^{11} s^{-1}\left(\tau_{\text {exp }}^{F R E T}=6.81 \mathrm{ps}\right)$. Consequently, we can conclude that the minimum energy geometry of the dimer is not optimal for energy harvesting by EET. These conclusions will inform future synthetic modifications to the structure to enhance EET efficiency.

We hypothesise that EET may also play a role in the unexpected slow bleaching which was observed in the dimer TA and EADS at $568 \mathrm{~nm}$. There is significant overlap between the ZnTPP fluorescence emission and the intense SubPc absorption $\left(J^{F}=4.127 \times 10^{-14} \mathrm{M}^{-1} \mathrm{~cm}^{-1} \mathrm{~nm}^{4}\right)$. Using Equation 5 to calculate the rate of the reverse energy transfer, and assuming that the orientational factor does not change between forward and backward EET, we obtain a slower time constant $k_{\text {calc }}^{\text {backRET }}=2.46 \times$ $10^{10} s^{-1}\left(\tau_{\text {exp }}^{\text {backRET }}=40.7 \mathrm{ps}\right)$. This suggests equilibration between forward and reverse energy transfer (the ZnTPP is also directly excited) in less than 100 ps. This is too fast for the observed effect, 
which occurs at T > $500 \mathrm{ps}$. Although, the ZnTPP steady-state emission spectrum (Figure 2 ) has a $Q(0,1)$ hot band which might be expected to have a shorter lifetime, compared to the relaxed $\mathrm{S}_{1}$ fluorescence, and therefore contribute less to the back EET. The contribution to the fluorescence spectrum of this hot band can be excluded by fitting the $Q(0,0)$ band of the ZnTPP emission with a Gaussian. The time constant associated with the back energy transfer process experiences a three-fold increase when we only consider the overlap between this relaxed fluorescence spectrum of ZnTPP and the absorption spectrum of the SubPc. This yields a relaxation time on the same order of magnitude as the unexpected bleach, but is clearly the slowest possible time that can be recovered assuming simple Förster transfer and the ground state structure. We speculate that this slow reverse relaxation may reflect a change in the structure after energy transfer, and that a complete explanation will then only be obtained through a coupled calculation of the time dependent excited state structure of the dimer and the geometry dependent energy transfer. All the parameters and the results for the FRET rate calculations are summarised in Table 2:

Table 2. Parameters used for the calculation of FRET rates (Equation 5) and results for forward and backward energy transfer

\begin{tabular}{|c|c|c|c|c|c|c|c|c|}
\hline & $\begin{array}{l}J^{F} \quad\left[M^{-1} \mathrm{~cm}^{-}\right. \\
\left.{ }^{1} \mathrm{~nm}^{4}\right]\end{array}$ & $\tau_{D}[\mathrm{ps}]$ & $\varphi_{D}$ & $\mathrm{~K}^{2}$ & $R(\AA ̊)$ & $n$ & $k_{\text {calc }}^{F R E T}\left[\mathrm{~s}^{-1}\right]$ & $\tau^{F R E T}[\mathrm{ps}]$ \\
\hline $\begin{array}{l}\text { SubPc } \\
\rightarrow \text { ZnTPP }\end{array}$ & $4.617 \mathrm{E}-14$ & $2.1 \mathrm{E} 3$ & 0.25 & 0.218 & 10.1 & 1.5 & $1.88 \mathrm{E} 11$ & 5.32 \\
\hline $\begin{array}{l}\text { ZnTPP } \quad \rightarrow \\
\text { SubPc }\end{array}$ & 4.127E-14 & $1.8 \mathrm{E} 3$ & 0.03 & 0.218 & 10.1 & 1.5 & 2.46E10 & 40.7 \\
\hline $\begin{array}{l}\text { ZnTPP } \rightarrow \\
\text { SubPc }\end{array}$ & $1.163 \mathrm{E}-14$ & $1.8 \mathrm{E} 3$ & 0.03 & 0.218 & 10.1 & 1.5 & $6.93 \mathrm{E} 9$ & 144 \\
\hline
\end{tabular}

${ }^{a}$ using the reduced emission spectrum (see text) 


\section{CLS analysis of the sub-ps dynamics}

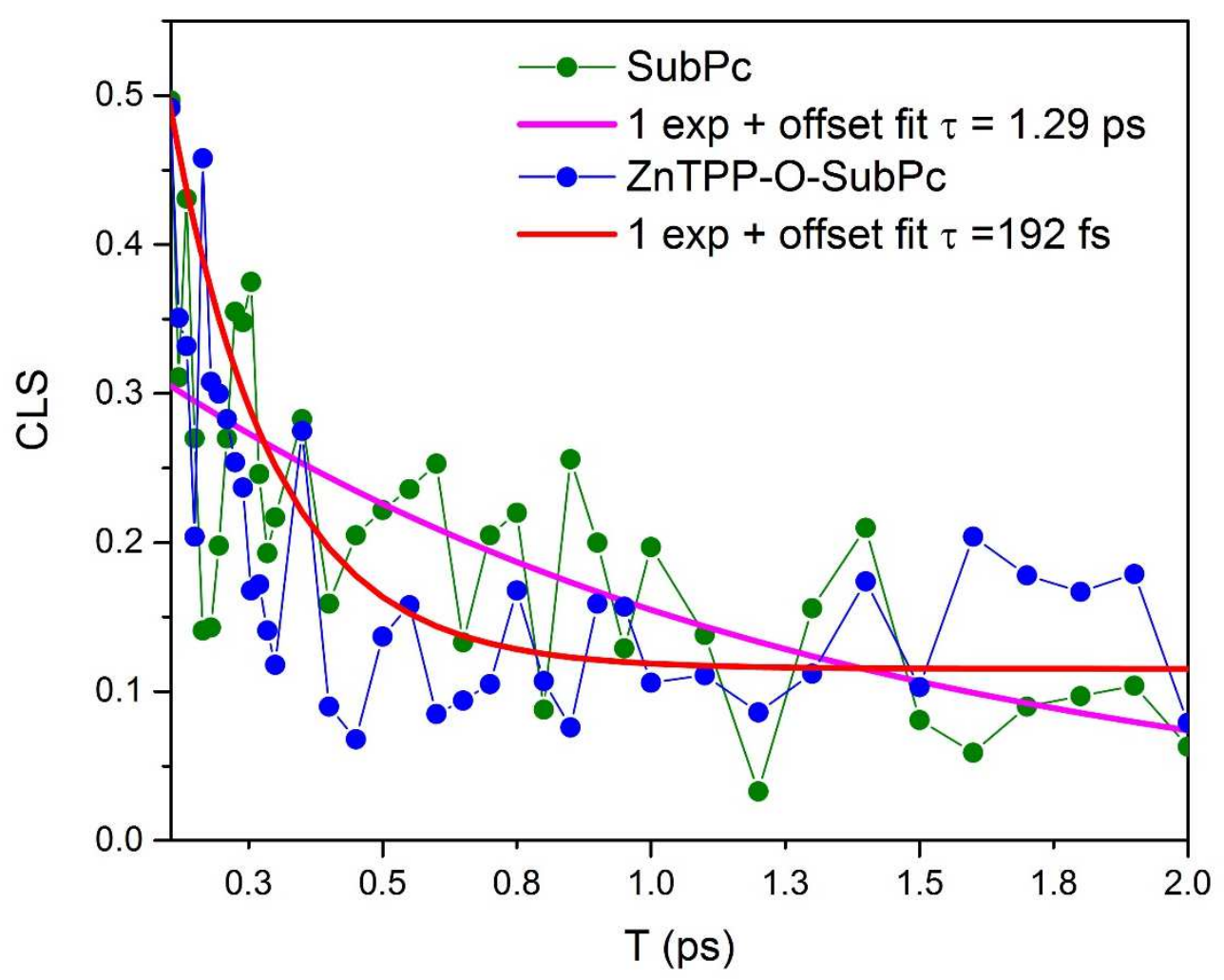

Fig. 9 extracted CLS values against population time T (75 fs to $2000 \mathrm{fs}$ ) for SubPc-Cl (green data, magenta fit) and ZnTPP-OSubPc (blue data, red fit).

Central line slope analysis in 2D-ES allows quantification of the energy gap fluctuations of the electronic transitions ${ }^{44}$; such fluctuations can be induced by electronic-vibrational or electronicsolvent interactions. Here the CLS analysis was carried out on the positive peaks of SubPc-Cl and on the ZnTPP-O-SubPc up to $T=2$ ps as shown in Figure 9. Both the SubPc-Cl (green dots) and ZnTPP-OSubPc (blue dots) CLS decay are dominated by sub-picosecond relaxation, although the signal-to-noise is poor (because of the non-Gaussian profile of the 2D peaks and the coexistence of positive and negative signals) making it difficult to extract more exact time constants for the spectral diffusion. For the dimer, the CLS reaches a plateau within $500 \mathrm{fs}$ and does not decay further, while the SubPc alone continues to relax towards zero. Thus these data point to fast spectral diffusion in both monomer and dimer, but the timescale is too fast to significantly impact upon the incoherent picosecond EET dynamics discussed above. 


\section{Conclusion}

We have investigated spectroscopy and energy transfer dynamics in a dyad comprising a strongly visible absorbing SubPc donor and a ZnTPP acceptor. The centre to centre distance between the two chromophores is small (ca $10 \AA ̊)$ but the spectra can be accurately reproduced assuming only weak dipole-dipole coupling, accompanied by combined Förster EET (on the timescale of a few picoseconds). The rate of EET is influenced by the dimer structure, which resulted in a relatively slow EET considering the short centre-to-centre distance. The picosecond energy transfer from the SubPc is followed by ISC in ZnTPP to populate the triplet state. An unexpected slow bleach of the SubPc, which may indicate a role for reverse EET accompanies these slower processes. Both fSTA and 2D-ES indicate the existence of subpicosecond dynamics, but these are present in both the donor and the heterodimer, and do not modify EET dynamics.

\section{Supporting Informations}

Calculated absorption and emission spectra of ZnTPP-O-SubPc, Decay Associated Difference Spectra from Glotaran fit of SubPc, ZnTPP and ZnTPP-O-SubPc, comparison between bleach traces of ZnTPP$\mathrm{OH}$ and ZnTPP-O-SubPc, spectral progression in the $0.1-10$ ps T interval for ZnTPP-O-SubPc and EET evidence from 2DES integrated time traces, sub-ps evolution of the 2D spectrum of ZnTPP-OH, DFT ground state structures and Transition Dipole Moments (from TD-DFT) of SubPc and ZnTPP in the dimer, DFT calculations for ZnTPP-O-SubPc, geometry optimisation and coordinates, Chemdraw structure of ZnTPP-O-SubPc.

\section{Acknowledgements}

The authors are grateful to EPSRC for financial support (EP/J009148/1 and J021431/1). GB is grateful to UEA for the award of a studentship. GB would like to thank Dr. K. Adamczyk for her help in setting 
up the fsTA experiment and D. Green for the helpful discussions. Calculations were performed on the High Performance Computing Cluster at the University of East Anglia.

\section{Bibliography}

(1) Gust, D.; Moore, T. A.; Moore, A. L. Molecular Mimicry of Photosynthetic Energy and Electron Transfer. Acc. Chem. Res. 1993, 26 (4), 198-205.

(2) Frischmann, P. D.; Mahata, K.; Würthner, F. Powering the Future of Molecular Artificial Photosynthesis with Light-Harvesting Metallosupramolecular Dye Assemblies. Chem. Soc. Rev. 2013, 42 (4), 1847-1870.

(3) Garner, R. K.; Josey, D. S.; Nyikos, S. R.; Dovijarski, A.; Wang, J. M.; Evans, G. J.; Bender, T. P. Boron Subphthalocyanines as Electron Donors in Outdoor Lifetime Monitored Organic Photovoltaic Cells. Sol. Energy Mater. Sol. Cells 2018, 176 (2017), 331-335.

(4) Kudisch, B.; Maiuri, M.; Blas-Ferrando, V. M.; Ortiz, J.; Sastre-Santos, A.; Scholes, G. SolventDependent Photo-Induced Dynamics in a Non-Rigidly Linked Zinc PhthalocyaninePerylenediimide Dyad Probed by Ultrafast Spectroscopy. Phys. Chem. Chem. Phys. 2017, 19, 21078-21089.

(5) Reid, O. G.; Pensack, R. D.; Song, Y.; Scholes, G. D.; Rumbles, G. Charge Photogeneration in Neat Conjugated Polymers. Chem. Mater. 2014, 26 (1), 561-575.

(6) Alfonso Hernandez, L.; Nelson, T.; Tretiak, S.; Fernandez-Alberti, S. Photoexcited Energy Transfer in a Weakly Coupled Dimer. J. Phys. Chem. B 2015, 119 (24), 7242-7252.

(7) Feng, L.; Rudolf, M.; Trukhina, O.; Slanina, Z.; Uhlik, F.; Lu, X.; Torres, T.; Guldi, D. M.; Akasaka, T. Tuning Intramolecular Electron and Energy Transfer Processes in Novel Conjugates of La 2 @C 80 and Electron Accepting Subphthalocyanines. Chem. Commun. 2015, 51 (2), 330-333.

(8) Balaban, T. S. Tailoring Porphyrins and Chlorins for Self-Assembly in Biomimetic Artificial Antenna Systems. Acc. Chem. Res. 2005, 38 (8), 612-623. 
(9) Günther, L. M.; Jendrny, M.; Bloemsma, E. A.; Tank, M.; Oostergetel, G. T.; Bryant, D. A.; Knoester, J.; Köhler, J. Structure of Light-Harvesting Aggregates in Individual Chlorosomes. J. Phys. Chem. B 2016, 120 (24), 5367-5376.

(10) Bolzonello, L.; Fassioli, F.; Collini, E. Correlated Fluctuations and Intraband Dynamics of JAggregates Revealed by Combination of 2DES Schemes. J. Phys. Chem. Lett. 2016, 4996-5001.

(11) Sprafke, J. K.; Kondratuk, D. V.; Wykes, M.; Thompson, A. L.; Hoffmann, M.; Drevinskas, R.; Chen, W. H.; Yong, C. K.; Kaernbratt, J.; Bullock, J. E.; et al. Belt-Shaped $\pi$-Systems: Relating Geometry to Electronic Structure in a Six-Porphyrin Nanoring. J. Am. Chem. Soc. 2011, 133 (43), 17262-17273.

(12) Cremers, J.; Haver, R.; Rickhaus, M.; Gong, J. Q.; Favereau, L.; Peeks, M. D.; Claridge, T. D. W.; Herz, L. M.; Anderson, H. L. Template-Directed Synthesis of a Conjugated Zinc Porphyrin Nanoball. J. Am. Chem. Soc. 2018, 140 (16), 5352-5355.

(13) Algethami, N.; Sadeghi, H.; Sangtarash, S.; Lambert, C. J. The Conductance of Porphyrin-Based Molecular Nanowires Increases with Length. Nano Lett. 2018, 18 (7), 4482-4486.

(14) Abdulalmohsin, S.; Cui, J. B. Graphene-Enriched P3HT and Porphyrin-Modified ZnO Nanowire Arrays for Hybrid Solar Cell Applications. J. Phys. Chem. C 2012, 116 (17), 9433-9438.

(15) Birel, Ö.; Nadeem, S.; Duman, H. Porphyrin-Based Dye-Sensitized Solar Cells (DSSCs): A Review. J. Fluoresc. 2017, 27 (3), 1075-1085.

(16) Chen, Y.; Li, A.; Huang, Z.-H.; Wang, L.-N.; Kang, F. Porphyrin-Based Nanostructures for Photocatalytic Applications. Nanomaterials 2016, 6 (3), 51.

(17) Liao, P.; Hu, Y.; Liang, Z.; Zhang, J.; Yang, H.; He, L. Q.; Tong, Y. X.; Liu, J. M.; Chen, L.; Su, C. Y. Porphyrin-Based Imine Gels for Enhanced Visible-Light Photocatalytic Hydrogen Production. J. Mater. Chem. A 2018, 6 (7), 3195-3201.

(18) Gouterman, M. Spectra of Porphyrins. J. Mol. Spectrosc. 1961, 6 (C), 138-163. 
(19) Baskin, J. S.; Yu, H. Z.; Zewail, A. H. Ultrafast Dynamics of Porphyrins in the Condensed Phase: I. Free Base Tetraphenylporphyrin. J. Phys. Chem. A 2002, 106 (42), 9837-9844.

(20) Yu, H. Z.; Baskin, J. S.; Zewail, A. H. Ultrafast Dynamics of Porphyrins in the Condensed Phase: II. Zinc Tetraphenylporphyrin. J. Phys. Chem. A 2002, 106 (42), 9845-9854.

(21) Cole, J. R.; Halas, N. J. Optimized Plasmonic Nanoparticle Distributions for Solar Spectrum Harvesting. Appl. Phys. Lett. 2006, 89 (15), 28-31.

(22) Gonzalez-Rodríguez, D.; Torres, T.; Olmstead, M. M.; Rivera, J.; Herranz, M. Á.; Echegoyen, L.; Castellanos, C. A.; Guldi, D. M. Photoinduced Charge-Transfer States in SubphthalocyanineFerrocene Dyads. J. Am. Chem. Soc. 2006, 128 (33), 10680-10681.

(23) Lissau, J. S.; Viñas Muñoz, A.; Gotfredsen, H.; Jevric, M.; Nielsen, M. B.; Sølling, T. I. Conformational Impact on Energy Storage Efficiency of Subphthalocyanine-Fullerene Hybrids. J. Phys. Chem. A 2018, 122 (33), 6683-6692.

(24) Munoz, A. V.; Gotfredsen, H.; Jevric, M.; Kadziola, A.; Hammerich, O.; Nielsen, M. B. Synthesis and Properties of Subphthalocyanine-Tetracyanobutadiene-Ferrocene Triads. J. Org. Chem. $2018,83(4), 2227-2234$.

(25) Winterfeld, K. A.; Lavarda, G.; Guilleme, J.; Sekita, M.; Guldi, D. M.; Torres, T.; Bottari, G. Subphthalocyanines Axially Substituted with a Tetracyanobuta-1,3-Diene-Aniline Moiety: Synthesis, Structure, and Physicochemical Properties. J. Am. Chem. Soc. 2017, 139 (15), 55205529.

(26) Azarias, C.; Pawelek, M.; Jacquemin, D. Structural and Optical Properties of Subporphyrinoids: A TD-DFT Study. J. Phys. Chem. A 2017, 121 (22), 4306-4317.

(27) Fulford, M. V.; Jaidka, D.; Paton, A. S.; Morse, G. E.; Brisson, E. R. L.; Lough, A. J.; Bender, T. P. Crystal Structures, Reaction Rates, and Selected Physical Properties of HaloBoronsubphthalocyanines (Halo = Fluoride, Chloride, and Bromide). J. Chem. Eng. Data 2012, 
$57(10), 2756-2765$.

(28) Claessens, C. G.; González-Rodríguez, D.; Torres, T. Subphthalocyanines: Singular Nonplanar Aromatic Compounds - Synthesis, Reactivity, and Physical Properties. Chem. Rev. 2002, 102 (3), 835-853.

(29) Osuka, A.; Bekki, Y.; Shimizu, D.; Fujimoto, K. Meso-Functionalizations of BIII Subporphyrin with BIII Meso-Lithiosubporphyrin. Chem. - A Eur. J. 2018, 24 (48), 12708-12715.

(30) Morse, G. E.; Castrucci, J. S.; Helander, M. G.; Lu, Z. H.; Bender, T. P. PhthalimidoBoronsubphthalocyanines: New Derivatives of Boronsubphthalocyanine with Bipolar Electrochemistry and Functionality in OLEDs. ACS Appl. Mater. Interfaces 2011, 3 (9), 35383544.

(31) Helander, M. G.; Morse, G. E.; Qiu, J.; Castrucci, J. S.; Bender, T. P.; Lu, Z. H. Pentafluorophenoxy Boron Subphthalocyanine as a Fluorescent Dopant Emitter in Organic Light Emitting Diodes. ACS Appl. Mater. Interfaces 2010, 2 (11), 3147-3152.

(32) Del Rey, B.; Keller, U.; Torres, T.; Rojo, G.; Agulló-López, F.; Nonell, S.; Martí, C.; Brasselet, S.; Ledoux, I.; Zyss, J. Synthesis and Nonlinear Optical, Photophysical, and Electrochemical Properties of Subphthalocyanines. J. Am. Chem. Soc. 1998, 120 (49), 12808-12817.

(33) Managa, M.; Mack, J.; Gonzalez-Lucasb, D.; Remiro-Buenamañana, S.; Tshangana, C.; Cammidge, A. N.; Nyokong, T. Photophysical Properties of Tetraphenylporphyrinsubphthalocyanine Conjugates. J. Porphyr. Phthalocyanines 2016, 20 (14), 1-20.

(34) Megerle, U.; Pugliesi, I.; Schriever, C.; Sailer, C. F.; Riedle, E. Sub-50 Fs Broadband Absorption Spectroscopy with Tunable Excitation: Putting the Analysis of Ultrafast Molecular Dynamics on Solid Ground. Appl. Phys. B Lasers Opt. 2009, 96 (2-3), 215-231.

(35) Hall, C. R.; Conyard, J.; Heisler, I. A.; Jones, G.; Frost, J.; Browne, W. R.; Feringa, B. L.; Meech, 
S. R. Ultrafast Dynamics in Light-Driven Molecular Rotary Motors Probed by Femtosecond Stimulated Raman Spectroscopy. J. Am. Chem. Soc. 2017, 139 (21), 7408-7414.

(36) Heisler, I. A.; Moca, R.; Camargo, F. V. A.; Meech, S. R. Two-Dimensional Electronic Spectroscopy Based on Conventional Optics and Fast Dual Chopper Data Acquisition. Rev. Sci. Instrum. 2014, $85(6), 63030$.

(37) Brixner, T.; Mančal, T.; Stiopkin, I. V.; Fleming, G. R. Phase-Stabilized Two-Dimensional Electronic Spectroscopy. J. Chem. Phys. 2004, 121 (9), 4221-4236.

(38) Harriman, A.; Porter, G.; Searle, N. Reversible Photo-Oxidation of Zinc Tetraphenylporphine by Benzo-1,4-Quinone. J. Chem. Soc. Faraday Trans. 2 Mol. Chem. Phys. 1979, 75, 1515-1521.

(39) El-Khouly, M. E. Electron Transfer Reaction of Light Harvesting Zinc NaphthalocyanineSubphthalocyanine Self-Assembled Dyad: Spectroscopic, Electrochemical, Computational, and Photochemical Studies. Phys. Chem. Chem. Phys. 2010, 12 (39), 12746-12752.

(40) Łapok, Ł.; Obłoza, M.; Solarski, J.; Pędziński, T.; Nowakowska, M. The Facile Synthesis, Triplet State Properties and Electrochemistry of Hexaiodo-Subphthalocyanine. Chem. - A Eur. J. 2018, 24(64),17080-17090.

(41) McEwan, K. J.; Bourhill, G.; Robertson, J. M.; Anderson, H. L. The Nonlinear Optical Characterization of Meso-Substituted Porphyrin Dyes. 2000, 9 (4), 451-468.

(42) Walters, V. A.; De Paula, J. C.; Jackson, B.; Nutaitis, C.; Hall, K.; Lind, J.; Cardozo, K.; Chandran, K.; Raible, D.; Phillips, C. M. Electronic Structure of Triplet States of Zinc(II) Tetraphenylporphyrins. J. Phys. Chem. 1995, 99 (4), 1166-1171.

(43) Fidler, A. F.; Harel, E.; Long, P. D.; Engel, G. S. Two-Dimensional Spectroscopy Can Distinguish between Decoherence and Dephasing of Zero-Quantum Coherences. J. Phys. Chem. A 2012, $116(1), 282-289$.

(44) Šanda, F.; Perlík, V.; Lincoln, C. N.; Hauer, J. Center Line Slope Analysis in Two-Dimensional 
Electronic Spectroscopy. J. Phys. Chem. A 2015, 119 (44), 10893-10909.

(45) Moca, R.; Meech, S. R.; Heisler, I. A. Two-Dimensional Electronic Spectroscopy of Chlorophyll a: Solvent Dependent Spectral Evolution. J. Phys. Chem. B 2015, 119 (27), 8623-8630.

(46) Snellenburg, J. J.; Laptenok, S. P.; Seger, R.; Mullen, K. M.; van Stokkum, I. H. M. Glotaran : A Java -Based Graphical User Interface for the R Package TIMP. J. Stat. Softw. 2012, 49 (3).

(47) Van Stokkum, I. H. M.; Larsen, D. S.; Van Grondelle, R. Global and Target Analysis of TimeResolved Spectra. Biochim. Biophys. Acta - Bioenerg. 2004, 1657 (2-3), 82-104.

(48) Dale, R. E.; Eisinger, J.; Blumberg, W. E. The Orientational Freedom of Molecular Probes. The Orientation Factor in Intramolecular Energy Transfer. Biophys. J. 1979, 26 (2), 161-193.

\section{TOC graphic:}

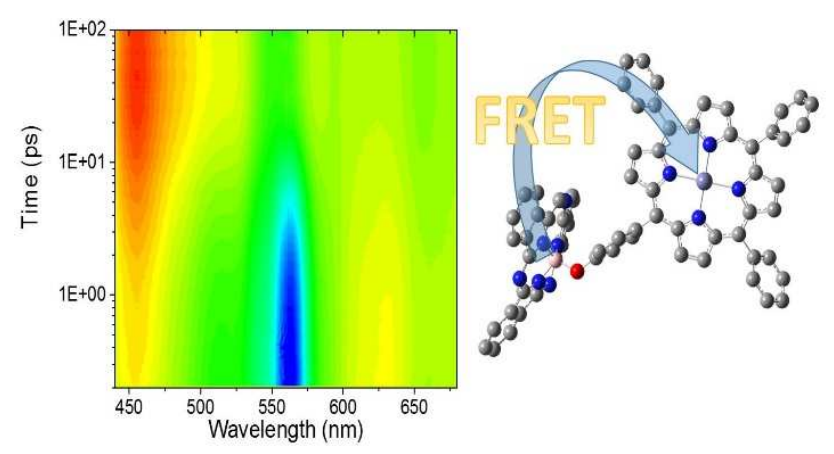

